\title{
Glutathione measurements in blood samples are influenced by oxygen saturation
}

\author{
Dieter Böning
}

Accepted: 31 August 2009 / Published online: 17 September 2009

(C) Springer-Verlag 2009

\section{Dear Editor}

Heinicke et al. (2009) describe a temporary increase in erythrocyte reduced glutathione concentration by $12-20 \%$ in venous blood samples after ascent to $2,800 \mathrm{~m}$ above the sea level. I wonder why the authors do not present also oxidized glutathione which yield additional insight since their method (Tietze 1969) allows determination of both forms. Also, they have difficulties to interpret their result as improved antioxidant defense, since the values return to control values within 2 weeks at altitude. The cause is probably that the changes are at least partly measuring artifacts: hemoglobin that binds glutathione much better in oxygenated than deoxygenated form (Craescu et al. 1986) is precipitated before analysis; thus, an unknown amount of glutathione disappears from the supernatant. We have shown that after in vitro equilibration with different oxygen pressures measured total glutathione concentrations decrease by $20 \%$ between 0 and $100 \% \mathrm{O}_{2}$ saturation (Hütler et al. 2000); the decrease in reduced glutathione is even larger (30\%), whereas the small fraction of oxidized glutathione increases fivefold. As venous oxygen content falls markedly during ascent and later rises again, this might be the cause of glutathione concentration changes. The possible error is easy to correct if the authors have measured venous $\mathrm{O}_{2}$ saturations. In addition, the change in concentrations of free reduced and oxidized glutathione within the red cell after a decrease in average $\mathrm{O}_{2}$ saturation should influence synthesis and trans-membrane transport of this substance. Therefore, all investigations without considering these effects do not allow clear conclusions about antioxidant defense by glutathione in blood.

\section{References}

Craescu CT, Poyart C, Schaeffer C, Garel MC, Kister J, Beuzard Y (1986) Covalent binding of glutathione to hemoglobin. II. Functional consequences and structural changes reflected in NMR spectra. J Biol Chem 261(31):14710-14716

Heinicke I, Boehler A, Rechsteiner T, Bogdanova A, Jelkmann W, Hofer M, Rawlings P, Araneda OF, Behn C, Gassmann M, Heinicke K (2009) Moderate altitude but not additional endurance training increases markers of oxidative stress in exhaled breath condensate. Eur J Appl Physiol 106:599-604

Hütler M, Pollmann C, Beneke R, Leithauser R, Böning D (2000) Measurable amount of glutathione in blood is influenced by oxygen saturation of hemoglobin. Clin Chim Acta 301:213-217

Tietze F (1969) Enzymic method for quantitative determination of nanogram amounts of total and oxidized glutathione. Anal Biochem 27:502-522
D. Böning $(\triangle)$

Sportmedizin Charité, Universitätsmedizin Berlin,

Campus Benjamin Franklin, Arnimallee 22,

14195 Berlin, Germany

e-mail: dieter.boening@charite.de 